\title{
System Architecture of the Dark Energy Survey Camera Readout Electronics
}

\author{
Theresa Shaw* $^{*}$, Otger Ballester ${ }^{\mathrm{b}}$, Laia Cardiel-Sas ${ }^{\mathrm{b}}$, Javier Castilla ${ }^{\mathrm{c}}$, Steve Chappa ${ }^{\mathrm{a}}$, \\ Juan de Vicente ${ }^{c}$, Scott Holmª, Dave Huffman ${ }^{\mathrm{a}}$, Mark Kozlovsky ${ }^{\mathrm{a}}$, Gustavo Martínez , \\ Todd Moore $^{\mathrm{d}}$, Jamieson Olsen ${ }^{\mathrm{a}}$, Vaidas Simaitis ${ }^{\mathrm{d}}$, Walter Stuermer ${ }^{\mathrm{a}}$ \\ ${ }^{\mathrm{a}}$ Fermi National Accelerator Laboratory ${ }^{1}$, Box 500, Batavia, IL, USA 60510 \\ ${ }^{\mathrm{b}}$ Institut de Física d'Altes Energies (IFAE) .Edifici Cn, Universitat Autònoma de Barcelona (UAB), \\ E-08193 Bellaterra (Barcelona), Spain \\ ${ }^{\mathrm{c} C e n t r o ~ d e ~ I n v e s t i g a c i o n e s ~ E n e r g e ́ t i c a s, ~ M e d i o a m b i e n t a l e s ~ y ~ T e c n o l o ́ g i a s ~(C I E M A T), ~ A v d a . ~}$ \\ Complutense, 22 - 28040 (Madrid) \\ ${ }^{\mathrm{d}}$ University of Illinois - Urbana-Champaign, Urbana, IL, USA 61801
}

\begin{abstract}
The Dark Energy Survey makes use of a new camera, the Dark Energy Camera (DECam). DECam will be installed in the Blanco 4M telescope at Cerro Tololo Inter-American Observatory (CTIO). DECam is presently under construction and is expected to be ready for observations in the fall of 2011. The focal plane will make use of $622 \mathrm{Kx} 4 \mathrm{~K}$ and 12 2kx2k fully depleted Charge-Coupled Devices (CCDs) for guiding, alignment and focus. This paper will describe design considerations of the system; including, the entire signal path used to read out the CCDs, the development of a custom crate and backplane, the overall grounding scheme and early results of system tests.
\end{abstract}

Keywords: Dark Energy, CCD, readout, electronics, Blanco, CTIO, DECam

\section{INTRODUCTION}

The Front End Electronics for the Dark Energy Camera must read out a large array of CCDs. The DECam focal plane will consist of 62 science CCDs, 4 guide CCDs and 8 alignment CCDs. The science CCDs are 2kx4k devices, while the guide and alignment CCDs are $2 \mathrm{kx} 2 \mathrm{k}$ devices. The readout requirements are that the science and alignment CCDs will be read out together at a rate of $250 \mathrm{kpixels} / \mathrm{s}$ with $<15 \mathrm{e}^{-} \mathrm{rms}$ noise. The guide CCDs will be read out during the integration time of the science array.

Each CCD which is read out is provided with its own set of clock and bias signals. No sharing of signal path occurs between individual CCDs. This means that we must provide 14 clock signals and 5 bias signals for each device. Each CCD also provides two analogvideo outputs. To read out the full focal plane, we must supply 1036 (74x14) clocks, 370 (74x5) bias voltages and 148 (74x2) analog video signals.

A description of the signal paths between the CCDs and the readout system will be provided in the following sections.

The Monsoon readout system ${ }^{1}$ is the choice of the collaboration for use with CCD characterization teststands and a slightly modified version of Monsoon has been developed for production installation on DECam. Monsoon is a cratebased image acquisition system developed by the National Optical Astronomy Observatory (NOAO). It is an open source system available for community use. Because we used Monsoon in our characterization of CCDs for the R\&D phase of the DECam Project, we have the advantage of using the experience we gained working with the original Monsoon system towards the implementation of the production DECam readout system. Monsoon offers the advantages

\footnotetext{
*Tshaw@fnal.gov, 1-630-840-2478

${ }^{1}$ Work supported by the U.S. Department of Energy under contract No. DE-AC02-07CH11359.
} 
of being crate-based, relatively compact and low power. There is also an experience base upon which we may draw, as well as a substantial amount of software development upon which to build.

The Monsoon system consists of three types of boards. The Master Control Board ${ }^{2}$ is the system interface. It controls all backplane functions, such as reading and writing to registers, as well as sequencing the readout of the CCDs. It communicates with a host computer through a fiber optic interface cable. All data are also delivered through this optical interface.

A second type of Monsoon board being used in our CCD characterization testing is the 8-Channel CCD Acquisition Board $^{3}$. This board currently provides eight channels to digitize the CCD video outputs as well as supplying 32 bias voltages.

The third type of Monsoon board in use for CCD characterization is the Clock and Bias Board ${ }^{4}$. This board provides 32 clock outputs and 40 bias outputs. Each clock output has independently adjustable low and high rail values.

We decided that DES was best served by some customization of the Monsoon boards. These customizations will increase the channel density to meet our space constraints and improve the flexibility and reliability of the final system. The customized boards which constitute the DECam Monsoon system are described in following sections.

\section{CCDS AND THE FOCALPLANE}

The DES focal plane, shown in Figure 1, will consist of sixty-two 2k x 4k CCDs (15 $\mu \mathrm{m} \times 15 \mu \mathrm{m}$ pixels) arranged in a hexagon covering an imaging area of 3 sq. degrees. Smaller format $2 k \times 2 k$ CCDs are used for guiding (G), and focusing (F), and are located around the edges of the focal plane. We have selected the fully depleted, high-resistivity, 250 micron thick CCDs that have been designed and developed ${ }^{5}$ at the Lawrence Berkeley National Laboratory (LBNL).

CCDs are built into pedestal packages. As shown in Figure 2, a complete pedestal package consists of the CCD, the Aluminum Nitride (AIN) readout board, the AlN spacer and the Invar foot. A 37 pin nanominiature series connector is located in the center of the device and is accessed through a hole in the foot. A temporary shorting plug is inserted into the connector to provide ESD protection.

The AlN readout board is approximately the same size as the CCD sensor, but slightly narrower so that the edge wirebond pads on the CCD can be used to wirebond from the CCD to the AlN backing hybrid. The thickness of the hybrid is $\sim 1 \mathrm{~mm}$ to provide mechanical stiffness and to maintain flatness. All metallization is on the top surface and will be gold. Solder pads are located near the center of the hybrid for a 37-pin nanominiature series connector and for a temperature sensor.

The readout method we have chosen for the CCDs requires us to provide 14 clock signals and 5 bias voltages per CCD. A partial path for these signals and the two analog readout channels is provided via a kapton Cable which connects to our CCD package.

\section{THE KAPTON CABLE}

A kapton cable, shown in Figure 3, is used to carry the CCD signals between the CCD and the Vacuum Interface Board. Our kapton cable is built using a semi-rigid design. Two 8 layer printed circuit boards are built on either end of a 3-layer kapton cable. The two rigid end boards of the cable are used to plug into the Vacuum Interface Board and into the CCD pedestal package. The rigid portion of the kapton cable which plugs into the CCD pedestal package contains an active JFET source follower used to reduce the high driver impedance of the CCD video output amplifier. The rigid end of the cable, which plugs into the Vacuum Interface Board, contains a pre-amplifier which drives the video signal to the Monsoon electronics. On the flexible middle portion of the cable, see Figure 4, three routing layers are used. The outer layers of the kapton cable will serve as ground/shield layers for the inner layer which will carry all signals. The cable 
(shields and signals) will be divided into two parts, with roughly half of the cable used to carry clock signals and the other half used to carry bias voltages and the video signals. The cable is about 10 inches long.

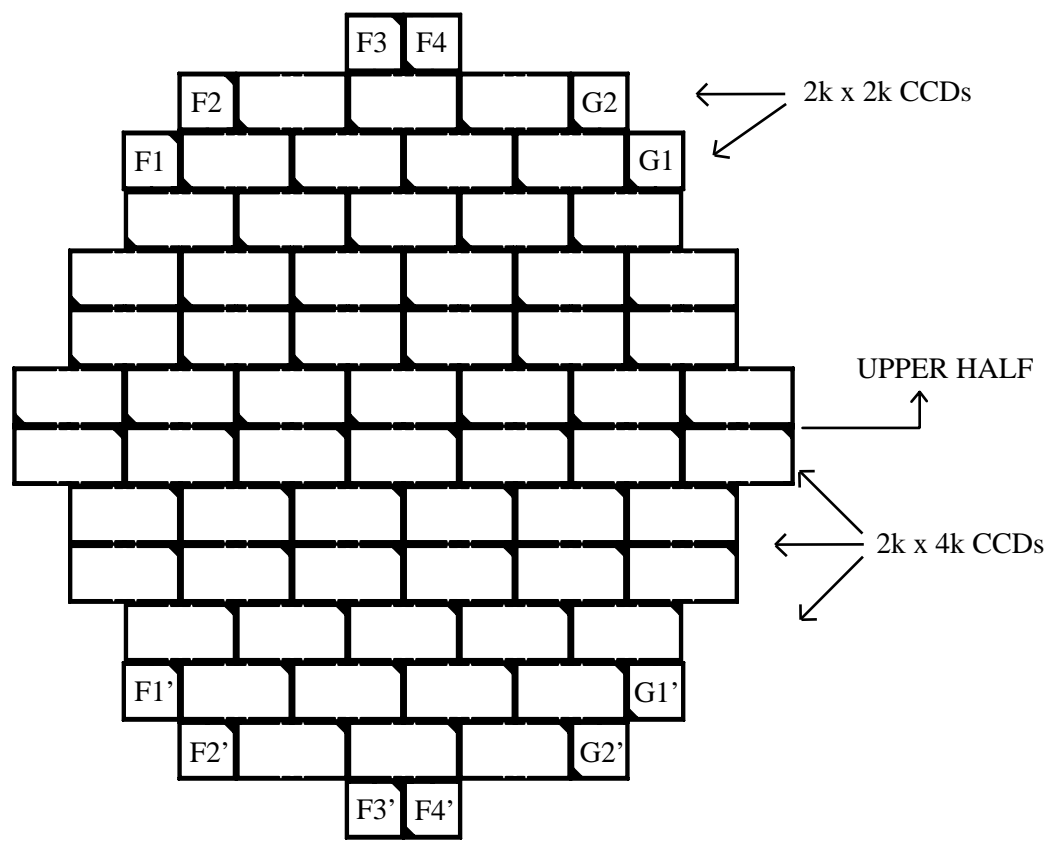

Figure 1. The DECam Focal plane is shown above. There are $622 \mathrm{kx} 4 \mathrm{k}$ CCDs. In the focal plane diagram, $\mathrm{G}$ labels the guide CCDs, and F labels the focus/alignment CCDs.

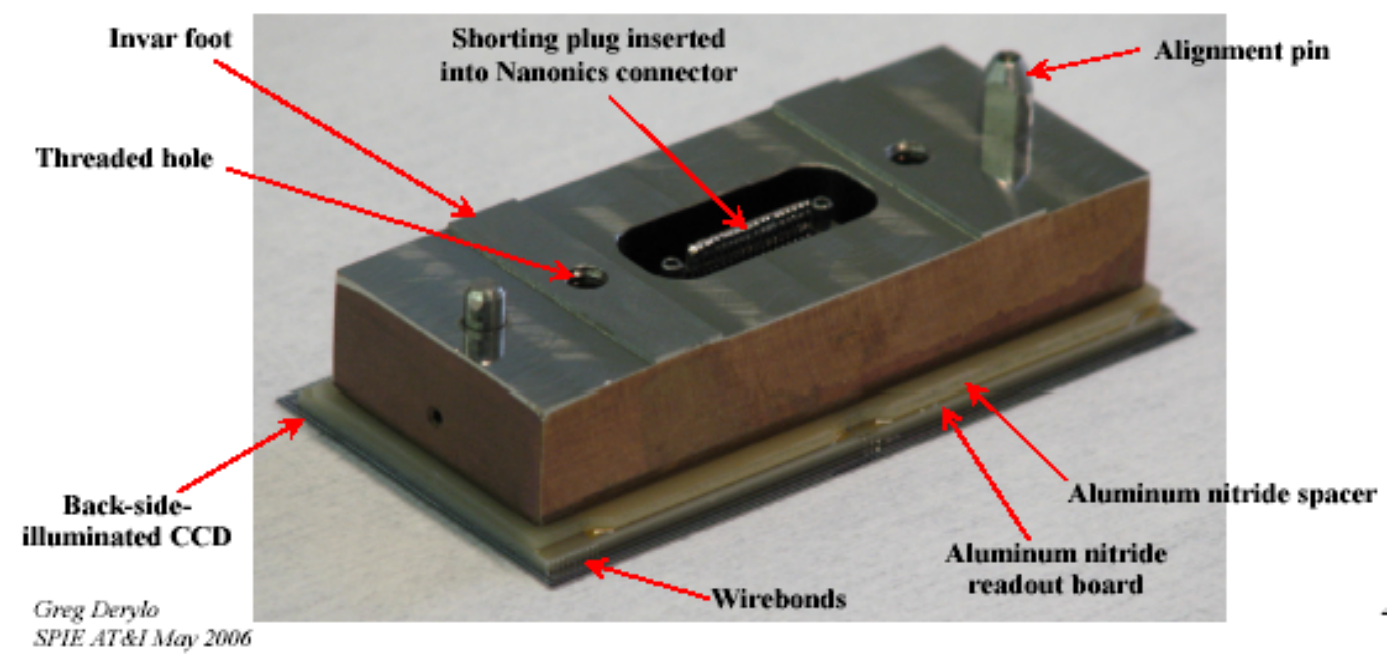

Figure 2. A complete pedestal package showing the CCD, the AlN readout board, the AlN spacer and the Invar foot. A 37 pin nanominiature connector is located in the center of the device and is accessed through a hole in the foot. A temporary shorting plug is inserted into the connector. 


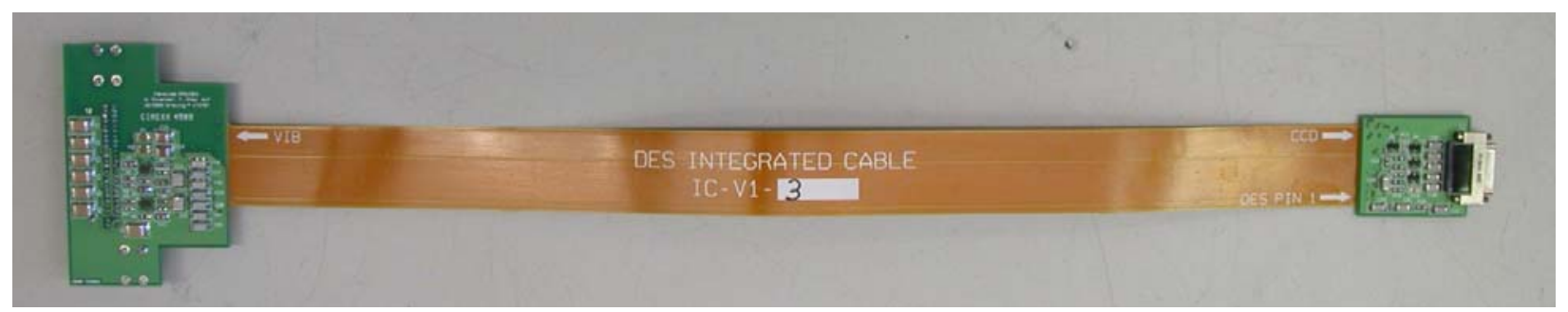

Figure 3. Semi-rigid Kapton cable. One end of the cable plugs into the Vacuum Interface Board and one end into the CCD pedestal package.

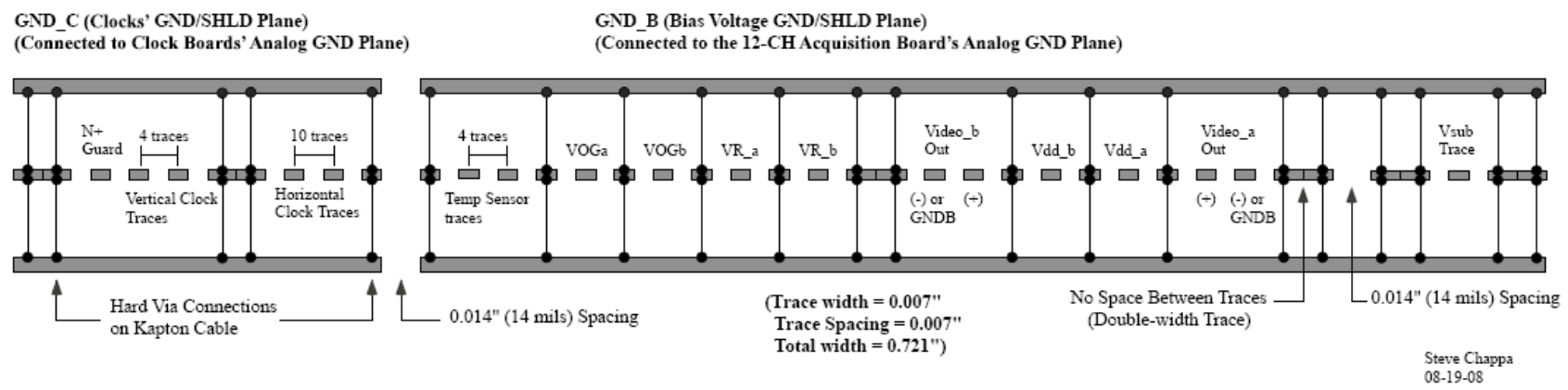

Figure 4. Illustration of the printed circuit construction of the flexible portion of the kapton cable.

\section{VACUUM INTERFACE BOARD}

Two Vacuum Interface Boards ${ }^{6}$ (VIBs) are used to route the clock, bias and video signals from the Monsoon readout electronics through the Dewar wall of the vacuum, see Figure 5. Once the signals are inside the Dewar, they are sent over Kapton cable assemblies to each CCD. The two vacuum interface boards bring a total of 2466 signals into the Decam Dewar.

The two VIB (East and West) assemblies penetrate the Dewar wall and are perpendicular to the wall. Each VIB is permanently epoxied to a vacuum flange, see Figure 6. An O-ring on the matching surface on the Dewar creates a vacuum seal. The VIB-WEST is a 28 layer board and is 26.7 ” $\mathrm{x} 12.2$ " in size (0.180" thickness). The VIB-EAST is $21.5 ”$ x $12.2 ”$ and 26 layers (0.155” thickness).

Figure 7 is a photograph of the inside our prototype test dewar. The kapton cables can be seen plugging into the VIB East and West.

Several features are incorporated into the VIB to preserve signal integrity. Cable shield layers on the top and bottom of the board are joined to form a continuous shielded box with a copper foil wrapped around the edge of the board and soldered to both sides. This serves to both to shield against external electrical noise and to block light that might otherwise make its way from the edge of the VIB to the inside of the vessel.

Copper foil is also used to form a near $360^{\circ}$ connection between the two shield layers and the vacuum flange. Finally, the vacuum flange forms a $360^{\circ}$ shield connection with the Dewar wall when the VIB is mounted in place. The cable shield layers do not enter the vessel. 


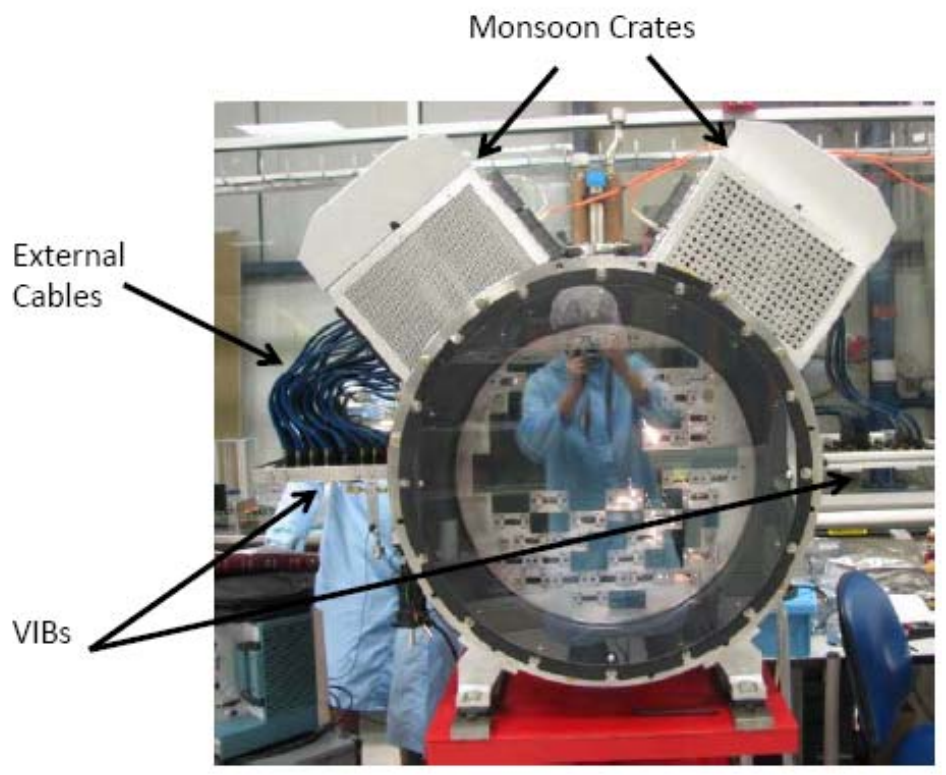

Figure 5. The Monsoon Crates mounted on a dewar, showing the external cables and Vacuum Interface Boards.

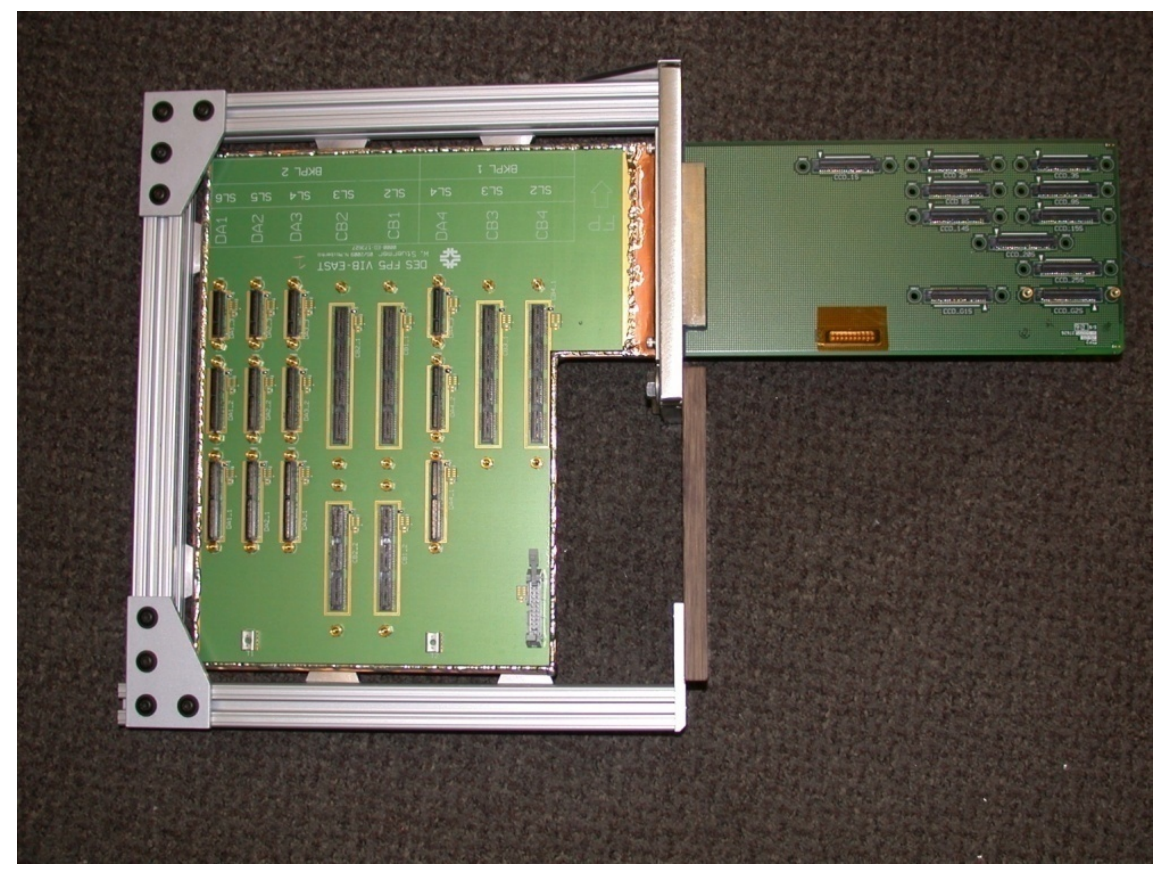

Figure 6 - VIB EAST witf flange and external support frame.

Signals on the VIB fall into 4 signal types: video, substrate bias, other CCD bias voltages, and CCD clocks. In fabrication of the printed circuit boards, signal types are put on different signals layers with bias ground or clock ground planes placed above and below each group. This is done to prevent crosstalk between video, clocks and bias signals. Analog video signals are routed for each CCD for quasi-differential readout. 


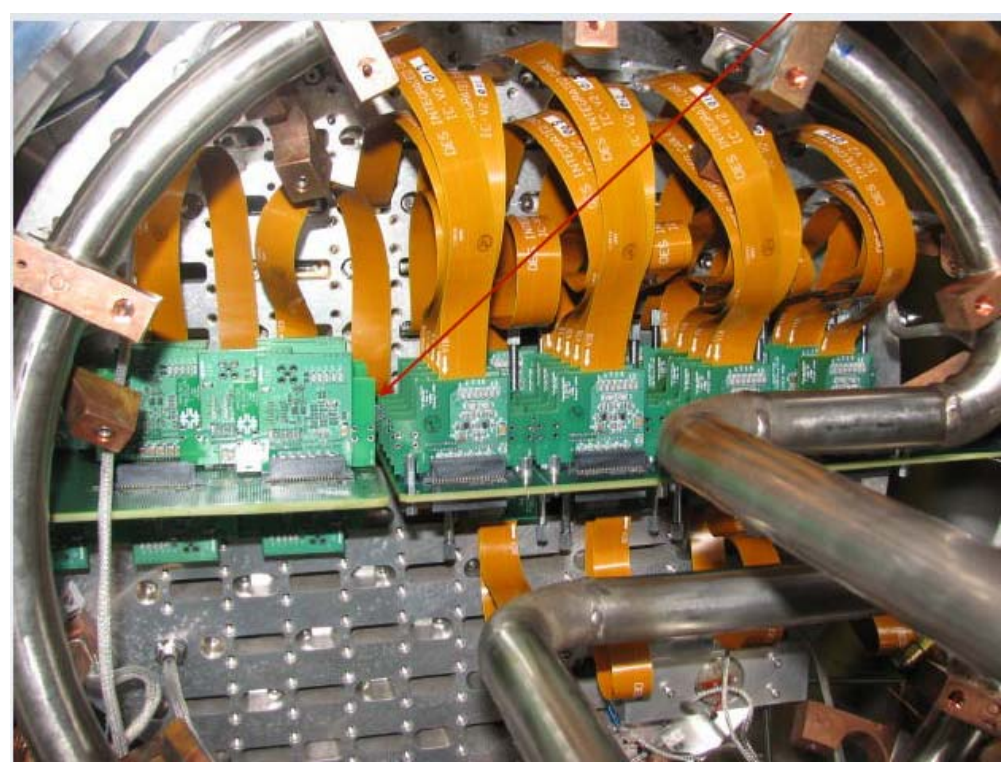

Figure 7 - Inside view of the dewar showing kapton cables plugging into the VIB.

Within each layer, signals are separated according to the clock or video card these signals go to. Bias and clock ground planes go back to the ground planes on the clock/video cards in the readout crate and are segmented within the VIB so as to avoid an undesirable connection between the analog ground planes on different cards.

The board stack-up for the VIB-EAST and WEST are shown in Figure 8.

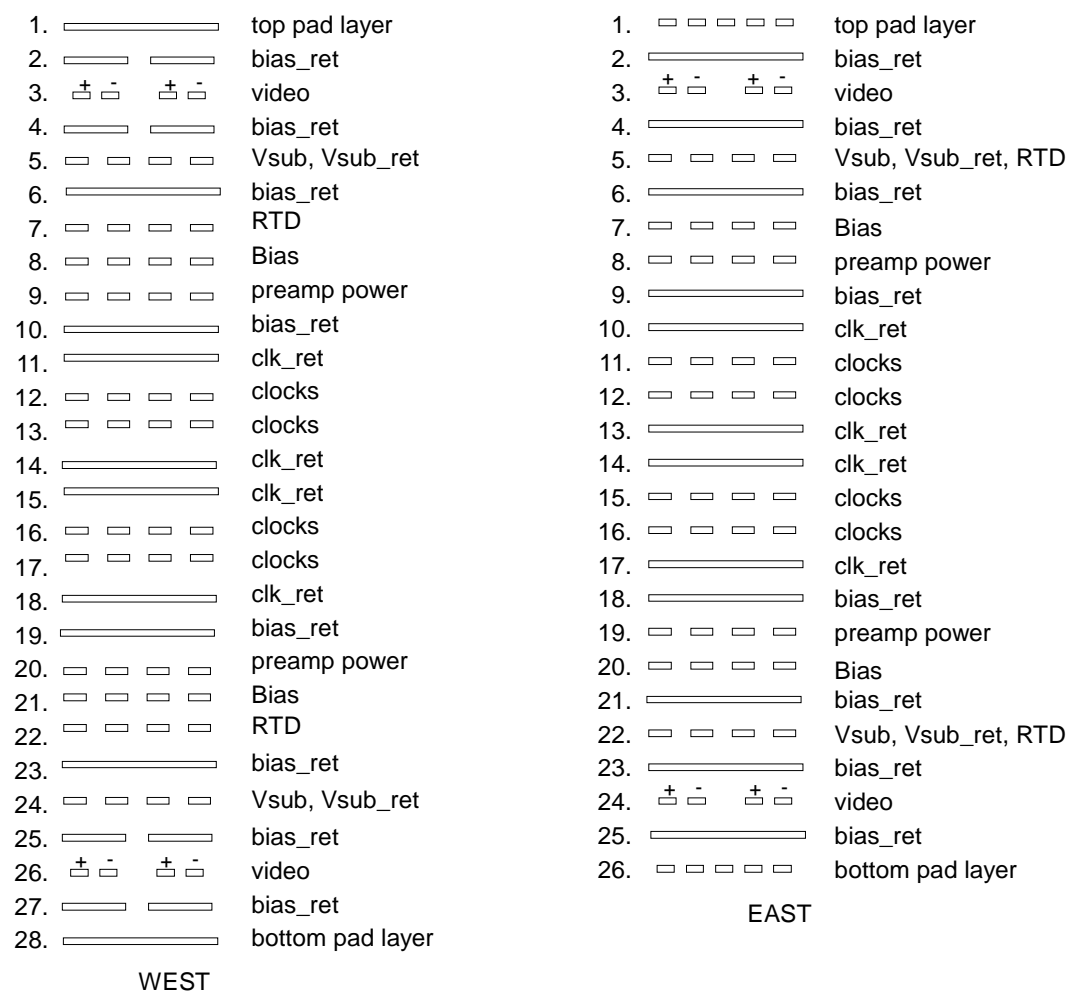

Figure 8 - PCB Board Stackups for VIB-EAST and VIB-WEST boards 


\section{EXTERNAL CABLES}

Two types of cabling are used to route the signals between the Monsoon Crates and the VIB. The Clock Board uses a shielded cable with twinax pairs. Of each clock signal pair, one leg of the twinax carries signal and one carries ground.

Signals going between the 12 Channel data acquisition module and the VIB make use of shielded coax cables. Figure 9, below, shows an external cable run implemented on our large test dewar.

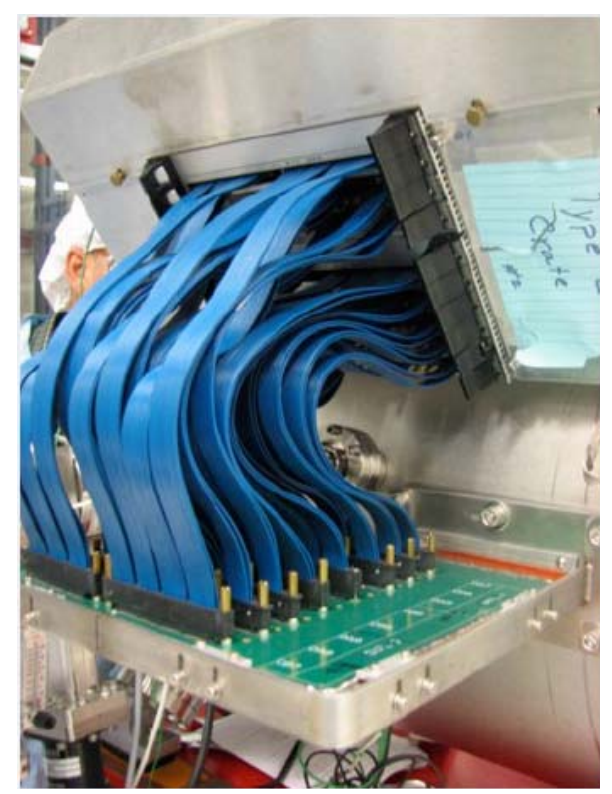

Figure 9. Photograph showing the External signal cables running between the VIB and the Monsoon Crate.

\section{DECAM ELECTRONICS CRATE}

\subsection{General Crate Description}

The DECam crate, shown in Figure 10, has dual 6-slot and 4-slot backplanes for a total of ten slots of main (front) Monsoon modules on the right side, and ten slots for $120 \mathrm{~mm}$ transition (rear) cards on the back side to the left. The transition cards get cabled to the Vacuum Interface Board (VIB) which connects them to the CCDs. At both ends of the crate there are air plenums to re-circulate the air through the Monsoon modules and transition cards. There is also an air plenum for the power supplies. There is a water-cooled heat exchanger at each end of the Monsoon modules, and two fans at each end of the transition cards. Two more fans at each end of the power supply plenum force some of the cooled air through the power supplies; the rest blows through the transition cards.

Separate DC power supplies will be used to power the fans, which must be powerful enough to overcome the pressure drops in the heat exchangers. The DC supplies will also power an independent internal crate monitor board.

The crate monitor board will communicate with the Instrument Control System ${ }^{7}$ (ICS) through opto-isolated signals. It will control and monitor the power supply power-up sequencing and shutdown, monitor all the power supply voltages, monitor the crate temperature, and monitor all fans for proper operation. 


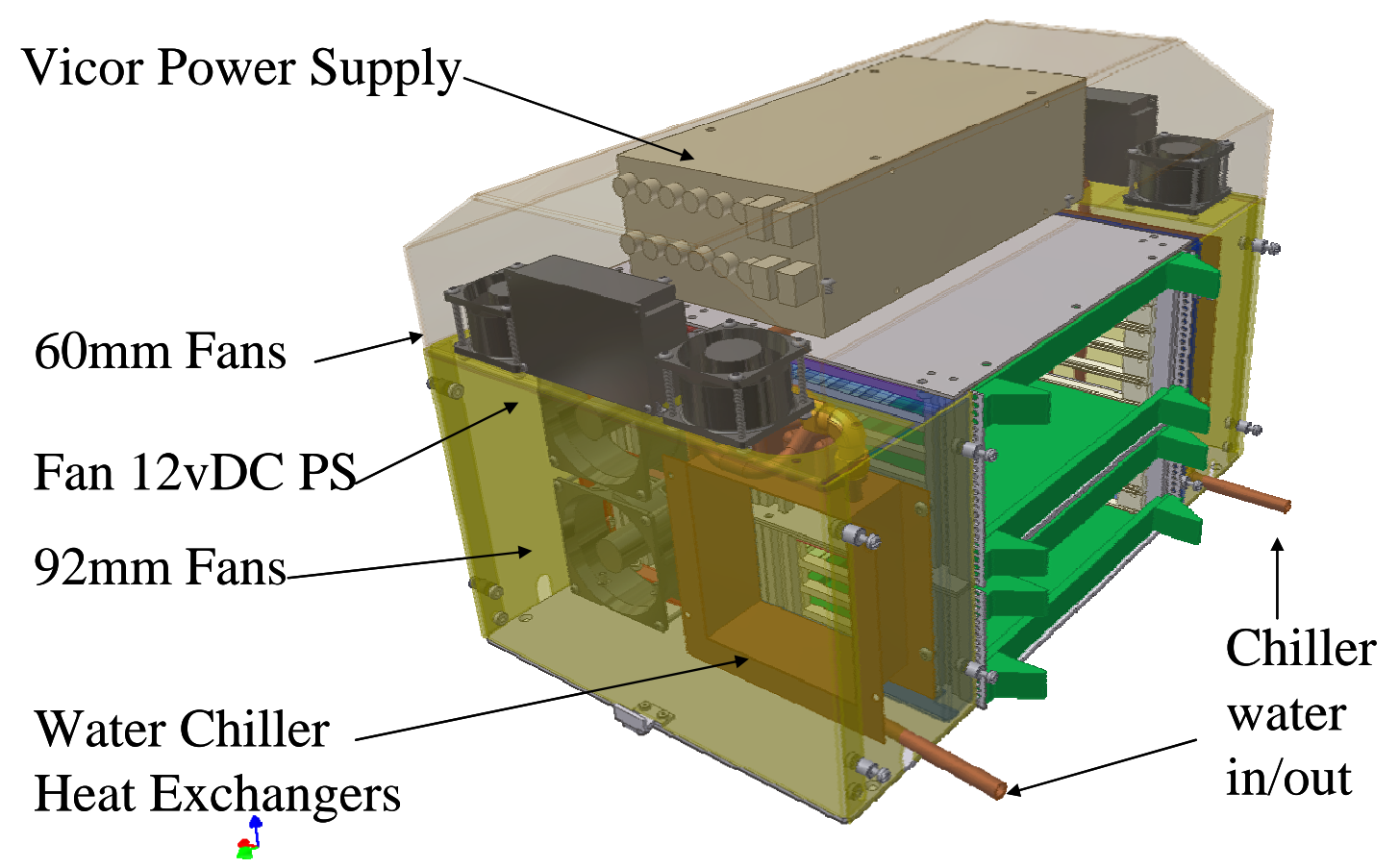

Figure 10. DECam Crate before heat shields are installed.

\subsection{Powering the Crates}

Early in the DECam crate design process it was determined that crate power would need to be in the form of a switching power supply. This determination was made primarily on the physical volume available for the power supply and the number of rail voltages required. A Vicor MegaPAC unit with 8 outputs was chosen as the solution. This did, however, require some additional filter circuitry to make it work.

Testing the acquisition system indicated the noise level emitted by the switching supply was higher than that of linear supplies and would need to be addressed. The outputs have several hundred millivolts of pk-pk noise at frequencies from $60 \mathrm{KHz}$ to $300 \mathrm{MHz}$. This noise has a large common mode level and a smaller differential level. Noise from the supply causes additional ADC noise counts in the acquisition channels and must be filtered out.

The Filter Box is designed to be part of the Low Voltage Power Supply for the Front End Electronics (FEE) and Heater Crates. The filter removes most of the switching noise produced by the commercial Vicor power supply. The Filter Box also provides a voltage monitor port and over temperature signal for the internal crate monitor board. The over temperature switch is set to close at $60^{\circ} \mathrm{C}$.

The FEE crates require eight voltage rails, two for digital and six for analog circuitry. The Heater crates require four rails, two for control circuits and two for power circuits. Each rail has its own filter and each filter is electrically identical. The filter uses feed-through capacitors for input and output connections. The filter box, see Figure 11, is a Faraday shield for the internal parts. The top, bottom and center layers of the circuit board act as EMI shields and will be connected to chassis ground. Each filter section or channel within the box has its own shield. The box dimensions are approximately 12"x6"x0.75". The box is mounted directly to the top of the Vicor power supply. The outputs of the Vicor connect directly to the inputs of the filter box. The output of the Filter Box connects directly to the analog backplane, distributing power to the Front End Electronics Transition cards. The analog backplane is a custom board installed parallel to the main backplane into which all transition boards plug. 
The filter is a differential/common mode filter and all components are rated to handle a minimum of 15 Amps continuous up to 100VDC.

Thermal management is handled by conduction from components to the PCB, then from secondary shields through thermal pads to the primary shield attached to the Vicor chassis.

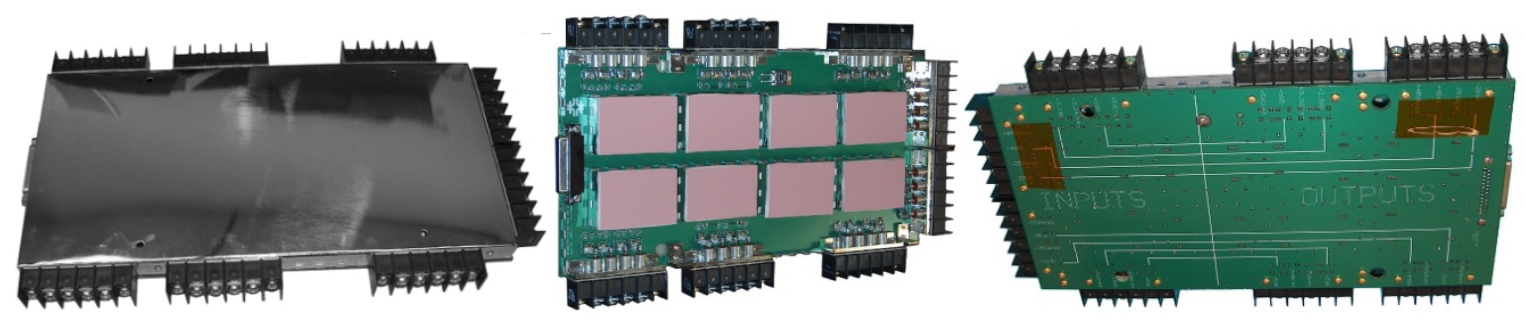

Figure 11. The custom filter box.

\subsection{Backplane Protocol}

DECam uses a proprietary backplane and data transfer protocol originally developed by NOAO for the MONSOON data acquisition system. While most read and write operations are controlled by the Master Control Board (MCB) the backplane protocol allows for multiple peripheral boards to arbitrate prioritized high-speed block transfers of pixel data through the MCB to the Pixel Access Node (PAN) computer.

The DECam backplane is synchronous to the rising edge of a $40 \mathrm{MHz}$ clock generated by the MCB. Each peripheral board slot receives a dedicated, independently-controlled buffered copy of the MCB system clock. For stand-alone operation, an internal oscillator on the MCB is used as the system clock. However, if precise system-wide clock synchronization is desired, the MCB has front panel $\mathrm{I} / \mathrm{O}$ and fine-grained delay adjustment hardware to support this mode of operation.

The MCB generates a separate board select signal for each slot while all other address, data, and control lines are bused to all boards. Two mode control lines define one of four types of bus access: 16-bit write, 32-bit write, 32-bit read, and board hard reset.

Pixel data transfers from the acquisition boards to the MCB require higher throughput than is currently available using discrete read cycles and thus a separate arbitration bus was created to facilitate high speed block transfers. In Pipeline Mode, each peripheral board is assigned a unique priority value. When an acquisition board has pixel data, it monitors the pipe_req_n bus and waits until all higher priority boards have completed their transfers. Then it drives the pixel data - two pixels at a time-on the 48-bit pix_data bus where it is written into a FIFO on the MCB and transferred immediately to the PAN computer over the fiber optic link. Burst mode is similar to Pipeline Mode except that the acquisition board holds on to the pixel data until explicitly told by the MCB to transmit the block of data. Both Pipeline and Burst transfer modes are effective, approaching the maximum throughput of $80 \mathrm{M}$ pixels per second.

\section{DECAM ELECTRONICS BOARDS}

\subsection{Introduction to DECam Electronics Boards}

The DECam Monsoon electronic boards closely follow the design of the original NOAO Monsoon boards with some important customizations specific to DECam requirements. The Master Control Board has been modified to use an open source optical communication link. The 8-Channel Acquisition Board will be redesigned to contain 12 channels and customized for our bias voltage needs. The Clock and Bias board will redesigned as a Clock Board capable of delivering 135 clocks rather than the 32 available in the original. These modifications are required in order for us to fit our electronics into the limited space available on the imager vessel. These changes will be described in more detail in subsequent sections. 
Like the original Monsoon system, all DECam Monsoon main modules have the format of a $6 \mathrm{U} 160 \mathrm{~mm} \mathrm{cPCI}^{8}$ card. All transition, or rear, modules have a $6 \mathrm{U} 120 \mathrm{~mm}$ format. Just as with the original Monsoon modules, a cPCI backplane is used; however most of the pin functions have been reassigned.

Figure 12 is a block diagram representing a 6-slot backplane which allows for the readout of up to 18 CCDs. This implementation makes use of a Master Control Board, two Clock Boards and three 12-Channel Acquisition Boards. DECam also makes use of a 4-slot backplane which contains a Master Control Board, one Clock Board and two 12Channel Acquisition Boards which can read out up to nine CCDs.

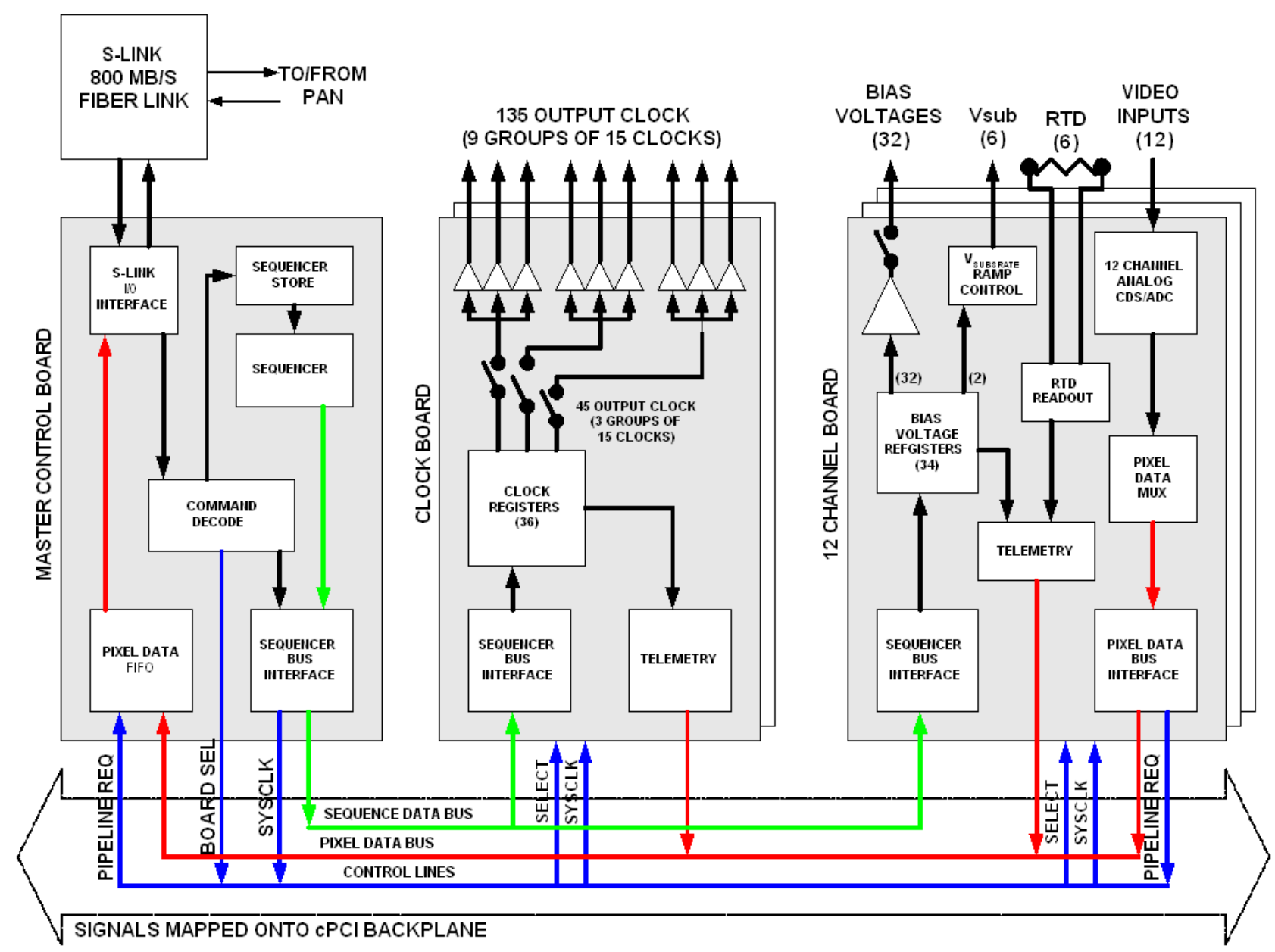

Figure 12. Block diagram of the DECam Monsoon system. All three board types have been re-designed and customized for DECam.

\subsection{2 Master Control Board}

The Master Control Board ${ }^{9}$ (MCB) acts as the bus master for the backplane bus and provides the interface between the peripheral boards in the Front End Electronics (FEE) crate and the Pixel Access Node (PAN) computer, which is connected via a bi-directional gigabit fiber optic link. Normally software running on the PAN computer sends a 
command the MCB, which performs a read or write operation on the backplane and optionally returns the requested data to the PAN. Repetitive operations such as CCD exposures benefit from using the onboard sequencer on the MCB.

Communication between the PAN computer and the MCB takes place over a fiber optic link called SLINK ${ }^{10}$. Originally developed at CERN for readout of the ATLAS particle detector systems, this fiber optic link format is proprietary and uses a custom PCI card (FILAR) in the PC and a custom mezzanine card (HOLA) on the MCB. At the most basic functional level the SLINK system can be best described as pair of 32-bit wide First-In First-Out memories (FIFOs). The PC writes data and commands into one FIFO which is read out and processed by the Field Programmable Gate Array (FPGA) on the MCB. In the other direction the MCB fills a FIFO and the data is transmitted to the PAN PC where it is placed directly into system memory by the FILAR card. A block diagram of the PAN - MCB system is shown in Figure 13.

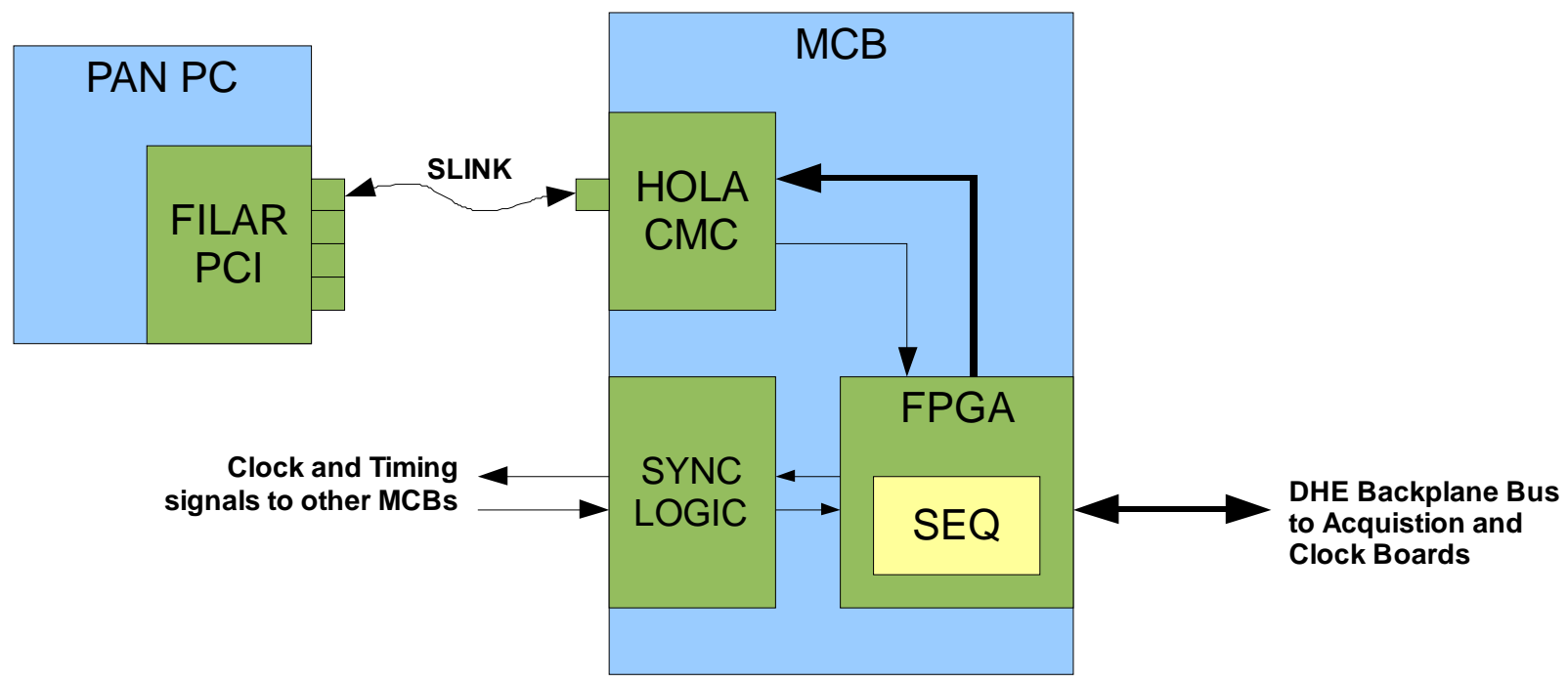

Figure 13. Master Control Board to PAN computer functional block diagram.

During normal data taking there are many repetitive operations taking place on the DHE backplane bus: the MCB writes to toggle CCD clock lines, other writes are to Acquisition board to control the pixel ADCs, etc. While these repetitive operations can be controlled directly from software on the PAN, the preferred method is to offload these tasks to a programmable sequencer in the MCB FPGA. The MCB sequencer programs are written in a type of macro assembly language which is then compiled on a PC and the binary image is downloaded into memories in the MCB FPGA. The sequencer assembly language features user variables, conditional branching as well as arithmetic functions. Loop count registers in the FPGA are readable and writable from the PAN as well as the sequencer code. Changing the values in the loop count registers thus modifies operation of the sequencer without the need to recompile and download - and this particularly useful when changing the size (or region of interest) of a CCD. The assembly language, assembler compiler and hardware interpreter is proprietary and was developed for the NOAO MONSOON system.

Since the DECam system is comprised of multiple crates, it is critical to provide a mechanism in hardware to synchronize operations across crates. A single MCB board is designated as the master and sends a copy of its $40 \mathrm{MHz}$ clock and a synchronization signal to a slave MCB board. Multiple slave MCBs may be daisy chained together. Adjustable digital delay lines and phase-shifting clock buffers are controlled by registers on the MCB board. The process of "dialing in" these delay parameters must be done once by hand in order to synchronize the system to nanosecond precision.

\subsection{Clock Board and Transition Modules}

The new DECam Clock Board ${ }^{11}$ (CB) has been designed to provide clocks for 9 CCDs and it includes 135 (9x15) clock outputs. In order to accomplish this, the CB design first creates 3 groups of 15 clock signals, or 45 clocks. Each of these 
45 clocks is fully programmable with independent control over the high and low voltage rails. We then take each of these 3 sets of 15 clocks and apply 3 output buffers to each signal for a total of 145 clock signals, or enough to drive clocks to 9 CCDs. In this manner, we lose the ability to control clock voltage levels on a CCD by CCD level, but maintain it at the level of a group of three CCDs. CCD characterization testing shows that it should not be difficult to group 3 or more CCDs together and have them operate optimally with the same level of clock settings.

The Clock Board Transition Module (CBT) plugs into the rear of the crate behind the Clock Board main module. The CBT is a simple module. Analog power for the Clock Board comes into this module, is filtered, and then passed to the Clock Board through unbussed backplane pins. The module also contains low pass filters, or waveshaping components, for each of the 135 clock outputs as well as the clock signal output connectors.

\subsection{Channel Board and Transition Modules}

The primary function of the 12-channel Acquisition ${ }^{12}$ module is to digitize the analog video signals from the CCDs and send this data over the backplane to the Master Control Board. Secondary functions include generating and reading back CCD bias voltages, monitoring temperatures, and storing calibration data. The block diagram of the 12-channel Acquisition board is shown in Figure 14.

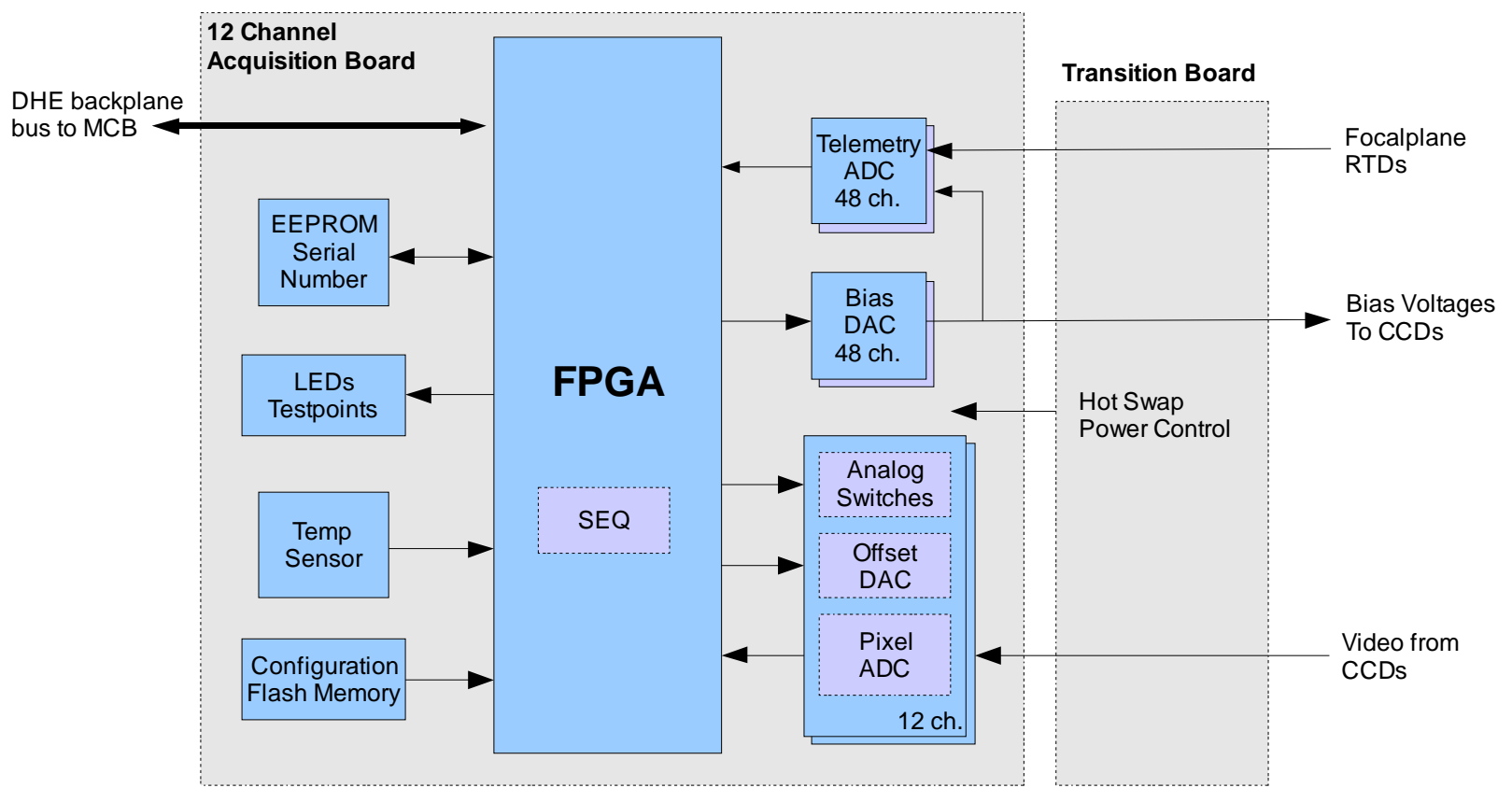

Figure 14. 12 Channel Acquisition functional block diagram.

Video signals from the CCDs are sent through a sensitive analog front end which performs correlated double sampling (CDS) of the video signal. The relatively complex analog circuitry used in the front end requires several analog switches which are controlled via digital signals from CDS registers in the FPGA. Pixel values are digitized with a fast 18-bit analog to digital converter (ADC) and stored in registers on the acquisition board before transmission back to the MCB and ultimately the PAN computer.

In addition to the analog front end circuits and ADCs, the acquisition board contains 60 independent digital to analog converter (DAC) channels for setting bias and offset voltages used by the CCD. The board also contains the readout electronics for the RTD temperature sensors located on the CCD pedestal packages. Each bias voltage DAC output is buffered and connected to a dedicated telemetry ADC channel for independent read back. Telemetry ADC channels may be individually configured for one of two gain settings and uni-polar or bi-polar operation. 
Configuration and calibration data may be stored directly on the acquisition board by utilizing the 4k-bit non-volatile EEPROM memory. This EEPROM also includes a unique 48-bit serial number which may be read from the backplane.

When the pixel ADCs complete their conversion cycle, the 18-bit values are stored in registers on the acquisition board. Only one set of pixel values may be stored on the acquisition board at a time. The MCB can read these registers directly using conventional backplane read cycles, however this is a relatively slow process. For better performance the acquisition board supports a pipeline data transfer mode, where the pixel values are quickly written to the MCB data FIFO at maximum speed without intervention immediately after ADC conversion completes. Pixel data bus arbitration amongst multiple acquisition cards is controlled by a priority scheme. Block transfers of pixel data are also supported in Burst Mode which is initiated by the MCB writing to a control register on each acquisition board. Redirection registers on the board specify which pixels are sent and in which order for each acquisition cycle.

In order to complete a single pixel acquisition cycle many operations must occur with a high degree of precision. While it is possible to control all of the low-level operations from the MCB, some of these repetitive tasks can be accomplished by using the sequencer in the acquisition board. The acquisition board sequencer is a finite state machine that when enabled, controls various CDS switches and ADC control signals. Each vector or state in this sequencer then has a delay parameter ranging from 25ns to 6.4 microseconds. A total of 64 16-bit vectors or states are stored in memory that is accessible from the backplane and may be read or written at any time. It is intended that the complex sequencer on the MCB will control the simple sequencers on the clock and acquisition boards to reduce the amount of backplane traffic needed to read out groups of CCDs.

The acquisition features a few useful debugging tools. The first is that all pixel ADC channels support the following test modes: all zeros, pseudorandom, and count-up. In the count-up test mode the pixel values are identified by the acquisition board number, physical channel number, and finally an index which counts from 0 to 99 and repeats. In this mode one can simply look at a pixel value to determine if the bus arbitration and software unpacking code is working properly. Another useful debugging tool is a backplane capture buffer which records up to 1024 backplane writes to the acquisition board. This capture buffer may be read out or cleared at any time.

The 12 channel transition board (rear module) is responsible for providing clean analog power to the acquisition board as well as filtering bias voltage outputs to the CCDs and receiving and buffering the video signal from the CCDs. Cable connections on the rear of the transition board go to the VIB on the imager vessel.

Analog power $(+48 \mathrm{~V},-28 \mathrm{v},+/-15 \mathrm{~V}$, and $+/-5 \mathrm{~V})$ is supplied over a small analog backplane located behind the main FEE crate backplane. The analog power rails are filtered and run through hot-swap controllers before passing through the main crate backplane to the acquisition board. Hot swap controllers insure that all analog voltages are stable and within specification before they are delivered to the acquisition board.

Bias voltages are filtered and clamped with a protection diode to insure they cannot damage the CCD. While generated on the acquisition board, the CCD substrate voltage has an applied gain and is filtered and buffered on the transition board before going to the CCDs.

\section{GENERAL GROUNDING AND SHIELDING}

\subsection{Grounding Introduction}

The DECam system grounding scheme, for discussion, is divided into three sections: 1) the grounding connections between the telescope structure and the cage assembly, 2) the grounding connections between the cage assembly and the DECam unit plus the grounding connections within the cage assembly, 3) the grounding connections between and within the various DECam camera components, right down to the individual PC board, cables, and CCDs. All grounding connections and shielding configurations are to be carefully controlled. The goals in each case are to 1) provide safety ground connections to all metallic components, 2) provide as "clean" as possible a zero voltage reference to these metallic components, and 3) protect the critical video signal path from noise sources. The term "clean" means that there should be no active or induced supply/return currents flowing through any chassis metallic. The guiding concept used to achieve these goals is to connect all of these components in a series of "star ground" configurations so that each metal 
component is connected to the grounding reference at only one point. Thus, this grounding scheme needs to place as much importance on the electrical isolation of various metallic components, return current conductors, and cable shields as on the dedicated electrical connections between these components.

\subsection{Telescope and Prime Focus Cage (PFC) Grounding Connections}

By original design, the entire PFC assembly is to be electrically isolated from the rest of the telescope and building's grounding configuration. This is done, however imperfectly, to simulate the "barbell" theory; an electrical enclosure connected to another electrical enclosure through a completely shielded connection(s). First, the electrical isolation at the mechanical attachment points (the location where the support fins attach to the cage's rib beams) will be accomplished by using G-10 fiberglass (or similar material with the necessary mechanical strength) insulating washers or plates. Secondly, all data communication for the DES readout and slow controls will be via optical links. Third, an isolation requirement, through the use of dielectric breaks or couplings, goes for any cooling or cryogenic metal tubing that goes from the building's telescope service center up to the cage assembly. These dielectric breaks or isolation couplings need to be placed as close to the cage assembly as possible. The reason for this is to prevent a long "antenna-like" conductor from being connected to the cage's metal or various components within the cage assembly and thus serve as a path for coupled lower frequency noise currents. Fourth, the cooling water/glycol mix should be low conductivity. Last, we do not allow any cables or copper conductors, originating off of the telescope, to electrically connect with the PFC assembly or its components. An exception is made for the hexapod cables. These cables are a special case that will need specific attention in order to adhere, as much as possible, to the desired grounding and shielding plan. Originally, the singular grounding connection between the building and the cage assembly metal was through a large (2/0 AWG) grounding cable. A separate power cable would then supply the cage's AC power needs. However, to simplify the cabling, a single shielded power cable will now be implemented to 1) supply the 3-phase AC power to the cage's power distribution chassis, and 2) provide both a safety ground connection (through the safety ground conductor) and a low impedance, high frequency ground connection (through the cable's shield braid). At the service end of this power cable, the grounding connection should be at the AC power distribution panel. This is to be the only connection between the building and/or telescope's grounded metal and the cage assembly's metal, thus ensuring that no large ground loop is formed between the cage assembly's metal and the building/telescope's ground structure. Also, in the unlikely event of an AC fault condition within the cage, the power cable, not the cage or telescope metal, will carry the fault current to the main breaker panel.

\subsection{Prime Focus Cage Components Grounding Scheme}

The grounding connections' purpose within the cage is to provide a single, controlled grounding connection between the DECam camera and the cage's star ground point. This is to minimize any conducted noise that could find its way to the critical signal paths within the camera. The point where the main AC power cable's shield braid attaches to the cage assembly's metal is at the AC power distribution chassis and this will serve as the cage's primary star ground point. Attached to this point are the grounding braids for the DECam camera (imager vessel, crates, etc.), the barrel assembly (lens housing, shutter, and filter changer), hexapod, and any components mounted on, but isolated from, the imager vessel. All of these flat grounding braids need to be short (no looping) and a width of 1-inch (minimum) to provide a solid, low-impedance path for any high frequency (HF) noise currents. Since the grounding connections are made using these braids only, it is important that these listed cage components' metal be electrically isolated from each other. Any metal to metal contact between the components' metal will defeat the controlled grounding connections and therefore provide possible conducted noise current paths as well as forming possible ground loops within the cage assembly.

\subsection{DECam Grounding Connections}

The DECam camera consists of the imaging vessel, the three front-end electronics (FEE) crates, and the focal plane support plate's heater crate. All of these listed components will be electrically isolated from each other. Ideally, the only electrical connections between these components' metal chassis and the imager vessel will be through dedicated grounding braids. Thus, the DECam will have its own star ground point. Here, the control of these connections, including any cable shield connections, is of the highest priority. Any conductive noise path to inside the dewar or to the electronics' analog video inputs within the front-end crates will directly and adversely affect the noise seen on these analog inputs and thus diminish the ability to achieve the readout noise below the maximum of $<15 \mathrm{e}^{-} \mathrm{rms}$. 


\subsection{The Critical Video Signal Path}

The final star ground point, and probably the most important, is on the CCD Interface (CCDI) board of the kapton cable. Complicating this configuration is fact that that the LBNL CCDs chosen do not have a ground reference plane as part of the active circuit. The CCDI star ground point cannot directly connect the ground reference planes used for the substrate voltage, the clock signals or the video signal and bias voltages. To do so would create potentially noisy ground loops since the clock signals and the video signal, along with the bias voltage signals, originate from different PC boards within a FEE crate. To confine a particular signal's return currents from interfering with other signals, separate ground reference planes are provided for the clock signals, the video signals and bias voltages, and the substrate voltage, from the CCDI board all the way to the FEE crate. Therefore, the connections to this CCDI star ground between the three ground reference planes are "soft", consisting of $0.01 \mathrm{uF}$ HF bypass capacitors. Since the video output signals are single-ended, high impedance signals, ground plane reference (inside the imager vessel) and cable shielding (outside the vessel) are critical. The vacuum interface board, on the "outside" section, contains an outside shield plane that is solidly bonded to the outside vessel wall. This shield plane, being solidly grounded to the vessel, serves as the video signals' and the FEE crates' ground reference and is where all signal cable shields are solidly connected.

\section{HEATER SYSTEM}

The Heater Controller Crate ${ }^{13}$ is used to help maintain a stable temperature at the focal plate within the Imager Dewar. It is capable of driving up to 12 heaters with internal resistance of 25 Ohms each. A heater requires up to 0.8 Amps of current or $20 \mathrm{~V}$ across it in order to keep focal plate within the required temperature range. Construction of the Heater Crate is similar to that of an FEE Crate but employs different set of VICOR power supplies.

A heater is driven by a differential driver which is controlled by a single-ended input signal of up to $10 \mathrm{~V}$ supplied by a National Instruments based Slow Control System. Differential output ensures an exact gain of 2, thus providing a maximum of $20 \mathrm{~V}$ across the heater. Driver output voltage is prevented from exceeding $24 \mathrm{~V}$ by employing a transient voltage suppressor with a clamp voltage of $12 \mathrm{~V}$ on both sides, negative and positive, of the driver. The latter is also protected from overheating by setting its maximum output current at 1.2A. If overheating occurs, the driver will shut itself down automatically and a warning signal will be provided via telemetry feedback to the Instrument Control System.

The very essential goal of the Heater Crate's design was to prevent heaters from introducing any additional noise into CCD readings. Various solutions were implemented towards that goal such as:

- Utilizing differential drivers to supply current to the heaters

- Heavy filtering of input and output signals

- Using isolation amplifiers to interface with the Slow Control System

- Employing separate sets of power supplies for the control and heater driver potions of the Heater Controller electronics

- Carefully designed grounding scheme in order to avoid any potentially hazardous ground loops

Extensive testing has proved effectiveness of those measures.

\section{CONCLUSION}

The DECam Readout Electronics are currently in production. This is after extensive testing of prototype modules and crates with our Multi-CCD Test Vessel. Over 20 engineering grade CCDs were instrumented and readout in that test vessel. We met our readout goals of better than $1 \%$ linearity and less than $<15 \mathrm{e}^{-}$rms noise at a readout rate of 250 kpixels/s.

We expect to be ready to install DECam in the Blanco 4M telescope at CTIO in early 2011 to be ready for observations in the fall. 


\section{ACKNOWLEDGEMENTS}

Funding for the DES Projects has been provided by the U.S. Department of Energy, the U.S. National Science Foundation, the Ministry of Science and Education of Spain, the Science and Technology Facilities Council of the United Kingdom, the Higher Education Funding Council for England, the National Center for Supercomputing Applications at the University of Illinois at UrbanaChampaign, the Kavli Institute of Cosmological Physics at the University of Chicago, Financiadora de Estudos e Projetos, Fundação Carlos Chagas Filho de Amparo à Pesquisa do Estado do Rio de Janeiro, Conselho Nacional de Desenvolvimento Científico e Tecnológico and the Ministério da Ciência e Tecnologia, the German Research Foundation-sponsored cluster of excellence “Origin and Structure of the Universe” and the Collaborating Institutions in the Dark Energy Survey.

The Collaborating Institutions are Argonne National Laboratories, the University of California at Santa Cruz, the University of Cambridge, Centro de Investigaciones Energeticas, Medioambientales y Tecnologicas-Madrid, the University of Chicago, University College London, DES-Brazil, Fermilab, the University of Edinburgh, the University of Illinois at Urbana-Champaign, the Institut de Ciencies de l'Espai (IEEC/CSIC), the Institut de Fisica d'Altes Energies, the Lawrence Berkeley National Laboratory, the Ludwig-

Maximilians Universität, the University of Michigan, the National Optical Astronomy Observatory, the University of Nottingham, the Ohio State University, the University of Pennsylvania, the University of Portsmouth, SLAC, Stanford University, and the University of Sussex.

\section{REFERENCES}

[1] Monsoon Image Acquisition System: http://www.noao.edu/ets/new_monsoon/MNSN_DataSheet_Rev1.0.pdf

[2] “Monsoon Master Control Board (MCB) Master Control Board Description”, P. Moore, NOAO Document MNSNAD-08-001 (March 2005).

[3] “Monsoon 8 Channel CCD Acquisition Board”, P. Moore, NOAO Document MNSN-AD-08-004 (September 2005).

[4] “Monsoon Clock and Bias Board”, P. Moore, NOAO Document MNSN-AD-08-003 (June 2006).

[5] "Improved spatial resolution in thick, fully depleted CCDs with enhanced red sensitivity”, S. Holland et al. IEEE Trans. Elec. Dev., 50, 225 (2003), and J. Fairfield et al., submitted to IEEE Trans. Nucl. Sci., (April 2006).

[6] “The Dark Energy Camera (DECam) Project Vacuum Interface Board Technical Design Report”, W. Stuermer, DECam 768 (February 2010).

[7] “DES ICS TDR 1.5.2.9”, J. Grudzinski, DECam Internal Note 792 (August 2007).

[8] Compact PCI Short Form Specification: http://www.picmg.org/test/pdf/cpcispec.pdf

[9] “MCB Developer Notes”, O. Ballester and L. Cardiel Sas, DECam Internal Note 4080 (January 2010).

[10] http://hsi.web.cern.ch/HSI/s-link/

[11] “Monsoon Clock Board”, J. Castilla , J. De Vincente, and G. Martinez, DECam Internal Note 1215 (June 2006 ).

[12] “Monsoon 12-Channel Acquisition Board Firmware and Register Definitions”, J. Olsen and D. Huffman, DECam Internal Note 354 (May 2010).

[13] “DECam Heater Controller System”, M. Kozlovsky, DECam Internal Note 3706 (October 2009). 